\title{
Hyperpolarized magnetic resonance shows that the anti- ischemic drug meldonium leads to increased flux through pyruvate dehydrogenase in vivo resulting in improved post- ischemic function in the diabetic heart
}

\author{
Dragana Savic $^{1,2}$ @ | Vicky Ball ${ }^{1}$ | Lorenz Holzner $^{3}$ | David Hauton ${ }^{1,4}$ | \\ Kerstin N. Timm ${ }^{1} \quad$ ～M. Kate Curtis ${ }^{1} \quad$ ～Lisa C. Heather ${ }^{1} \quad$ Damian J. Tyler ${ }^{1,2}$ (6)
}

${ }^{1}$ Cardiac Metabolism Research Group (CMRG), Department of Physiology, Anatomy and Genetics, University of Oxford, Oxford, UK

${ }^{2}$ Oxford Centre for Clinical Magnetic

Resonance Research (OCMR), Division of

Cardiovascular Medicine, Radcliffe

Department of Medicine, University of Oxford, Oxford, UK

${ }^{3}$ Department of Physiology, Development and Neuroscience, University of Cambridge,

Cambridge, UK

${ }^{4}$ Metabolomics Research Group, Department of Chemistry, University of Oxford, Oxford, UK

Correspondence

Dr Dragana Savic, Oxford Centre for Clinical Magnetic Resonance Research (OCMR), Division of Cardiovascular Medicine, Radcliffe Department of Medicine, University of Oxford, Oxford OX3 9DU, UK.

Email: dragana.savic@cardiov.ox.ac.uk

Funding information

BHF Senior Research Fellowship, Grant/Award Number: FS/17/58/33072; Danish Council for Strategic Research; NIHR Oxford Biomedical Research Centre; Novo Nordisk Postdoctoral Fellowship; OXFORD-BHF Centre for Research Excellence, Grant/Award Number: $\mathrm{RE} / 13 / 1 / 30181$
The diabetic heart has a decreased ability to metabolize glucose. The anti-ischemic drug meldonium may provide a route to counteract this by reducing L-carnitine levels, resulting in improved cardiac glucose utilization. Therefore, the aim of this study was to use the novel technique of hyperpolarized magnetic resonance to investigate the in vivo effects of treatment with meldonium on cardiac metabolism and function in control and diabetic rats. Thirty-six male Wistar rats were injected either with vehicle, or with streptozotocin $(55 \mathrm{mg} / \mathrm{kg})$ to induce a model of type 1 diabetes. Daily treatment with either saline or meldonium $(100 \mathrm{mg} / \mathrm{kg} / \mathrm{day})$ was undertaken for three weeks. in vivo cardiac function and metabolism were assessed with CINE MRI and hyperpolarized magnetic resonance respectively. Isolated perfused hearts were challenged with low-flow ischemia/reperfusion to assess the impact of meldonium on post-ischemic recovery. Meldonium had no significant effect on blood glucose concentrations or on baseline cardiac function. However, hyperpolarized magnetic resonance revealed that meldonium treatment elevated pyruvate dehydrogenase flux by 3.1-fold and 1.2-fold in diabetic and control animals, respectively, suggesting an increase in cardiac glucose oxidation. Hyperpolarized magnetic resonance further demonstrated that meldonium reduced the normalized acetylcarnitine signal by 2.1-fold in both diabetic and control animals. The increase in pyruvate dehydrogenase flux in vivo was accompanied by an improvement in post-ischemic function ex vivo, as meldonium elevated the rate pressure product by 1.3 -fold and 1.5 -fold in the control and diabetic animals, respectively. In conclusion, meldonium improves in vivo pyruvate dehydrogenase flux in the diabetic heart, contributing to improved cardiac recovery after ischemia.

Abbreviations: 3-OHB, 3-hydroxybutyrate; BPM, beats per minute; i.p., intraperitoneal; LC-MS, liquid chromatography-mass spectrometry; NEFA, non-esterified fatty acid; PDH, pyruvate dehydrogenase; RPP, rate pressure product; SD, standard deviation; STZ, streptozotocin; TAG, triglyceride; $T_{\mathrm{E}}$, echo time; $T_{\mathrm{R}}$, repetition time. 


\section{KEYWORDS}

cardiac function, diabetes, hyperpolarized MRI, langendorff perfusion, meldonium, metabolism, pyruvate dehydrogenase flux, streptozotocin

\section{1 | INTRODUCTION}

Diabetes has become increasingly prevalent in recent years, with a global incidence estimated to rise from 108 million people in 1980 to 700 million people by $2045 .{ }^{1}$ Of these people, it is suggested that $5-15 \%$ will have type 1 diabetes. ${ }^{2}$ Diabetes is associated with an elevated risk of cardiovascular disease and heart failure, ${ }^{3,4}$ and cardiovascular disease is the major cause of premature death in diabetic patients. ${ }^{5}$ Therefore, therapies that protect the heart in diabetes are urgently needed to reduce mortality.

Dysregulated cardiac substrate utilization and mitochondrial dysfunction are contributing factors to impaired cardiac function in the diabetic population. ${ }^{6,7}$ In the healthy heart, $60-70 \%$ of ATP production comes from fatty acids, and the remaining $30-40 \%$ comes from glucose, lactate and amino acids. However, in diseases such as diabetes, this balance is shifted even further in favor of fatty acid oxidation. ${ }^{8,9}$ Therefore, drugs that target this metabolic imbalance in the diabetic heart are of great therapeutic interest. One such drug is the anti-ischemic agent meldonium (trimethylhydrazinium propionate), which is sold under the brand name Mildronate. The anti-ischemic actions of meldonium are thought to originate from a switch in the balance of fuel utilization in the heart towards more oxygen-efficient glucose metabolism. These effects arise from the impact of meldonium on the synthesis and transport of the amino acid derivative L-carnitine.

L-carnitine is required for the transport of long-chain fatty acids into mitochondria via the carnitine shuttle. L-carnitine accepts the fatty acid moiety from long-chain acyl-CoAs found in the cytoplasm, making them a suitable substrate for mitochondrial uptake via the carnitineacylcarnitine translocase within the inner mitochondrial membrane. Meldonium has been shown to reduce L-carnitine availability both through inhibition of the L-carnitine transporter OCTN2, inhibiting absorption and reabsorption of L-carnitine in tissues, as well as through inhibition of the last step in the biosynthesis of L-carnitine, $\gamma$-butyrobetaine hydroxylase.

As such, treatment with meldonium can be hypothesized to reduce fatty acid uptake and oxidation in the mitochondrial matrix and provide a drive to increase glucose oxidation via the Randle cycle, ${ }^{10,11}$ primarily through reduced acetyl-CoA availability, which is known to inhibit glucose oxidation at the key regulatory step of pyruvate dehydrogenase (PDH) ${ }^{12}$ In a proof-of-concept study, we have shown previously that therapeutic strategies that improve mitochondrial pyruvate oxidation via increased PDH activity result in improved cardiac function in diabetes. ${ }^{13}$ However, clinically relevant compounds that improve cardiac PDH flux in diabetes are lacking. By modulating carnitine availability, meldonium may therefore provide a route to improve cardiac glucose utilization via increased $\mathrm{PDH}$ flux in diabetes.

This study aimed to investigate the effects of meldonium on in vivo cardiac metabolism and function in control and diabetic rodent hearts, using hyperpolarized MRS and CINE MRI. Hyperpolarized MRS is a novel technique, which can increase the in vivo sensitivity of MRS to detect

${ }^{13} \mathrm{C}$-labeled metabolic substrates by more than 10000 -fold. ${ }^{14}$ It therefore enables unprecedented real-time visualization of the biochemical mechanisms of normal and abnormal metabolism, with measurement of instantaneous substrate uptake and enzymatic transformation in vivo. ${ }^{15}$ Following the injection of ${ }^{13} \mathrm{C}$ pyruvate labeled at the first carbon position $\left(\left[1-{ }^{13} \mathrm{C}\right]\right.$ pyruvate), it is possible to see the metabolic production of carbon dioxide and bicarbonate $\left({ }^{13} \mathrm{CO}_{2}\right.$ and $\left.\mathrm{H}^{13} \mathrm{CO}_{3}{ }^{-}\right)$through the $\mathrm{PDH}$ enzyme complex, yielding a marker of oxidation from carbohydrate sources. In addition, following injection of pyruvate labeled with ${ }^{13} \mathrm{C}$ at the second carbon position $\left(\left[2-{ }^{13} \mathrm{C}\right]\right.$ pyruvate), it is possible to measure flux through the Krebs cycle, along with incorporation of acetyl-CoA into acetylcarnitine, providing a marker of L-carnitine availability. Hyperpolarized MRS therefore provides an ideal approach to explore the impact of meldonium treatment on the diabetic heart in vivo.

\section{2 | METHODS}

\subsection{Animal experimentation ethical approvals}

All procedures (i) had local approval and (ii) conformed to the guidelines from Directive 2010/63/EU of the European Parliament on the protection of animals used for scientific purposes or the NIH Guide for the Care and Use of Laboratory Animals. Animal studies were conducted in accordance with the UK Animals (Scientific Procedures) Act (1986), PPL Number 30/3322, and local ethical guidelines (Medical Research Council Responsibility in the Use of Animals for Medical Research, July 1993).

\section{2 | Anesthetic agents}

During all in vivo procedures, $2.5 \%$ isoflurane vol.:vol. in $1 \mathrm{~L} / \mathrm{min} \mathrm{O}_{2}$ was given via inhalation for anesthesia. An overdose of $5 \%$ isoflurane vol.: vol. in $2 \mathrm{~L} / \mathrm{min}_{2}$ was used for euthanasia; once the pedal and corneal reflexes ceased, the chest cavity was opened and the heart was excised. 


\section{3 | Protocol}

Thirty-six healthy male Wistar rats ( 200 g) were randomly divided into four groups. All animals were fasted overnight (food removed for 12-15 h) and then either made diabetic with one intraperitoneal (i.p.) injection of streptozotocin (STZ, $55 \mathrm{mg} / \mathrm{kg}$ ) or kept as controls via an injection of citrate buffer. Diabetes was confirmed by ensuring glucose levels higher than $110 \mathrm{mmol} / \mathrm{L}$ in urine samples using reagent test strips (Dirui, Jilin, China) for two consecutive days following STZ injection and by showing blood glucose levels higher than $8.3 \mathrm{mmol} / \mathrm{L}$ (Accu-Chek Aviva, Roche, Basel, Switzerland) at $6 \mathrm{~d}$ after STZ injection. Two weeks after STZ/citrate buffer injection, all animals were initiated on daily morning i.p. treatment with either saline or meldonium $(100 \mathrm{mg} / \mathrm{kg} /$ day). After two weeks of treatment, all animals were anesthetized with isoflurane and subjected to MRI and hyperpolarized MRS.

After three weeks of treatment, all animals were euthanized in the fed state with $5 \%$ isoflurane vol.:vol. in $2 \mathrm{~L} / \mathrm{min}_{2}$, followed by removal of the heart for Langendorff perfusion, with blood and tissue collected for analysis. Blood samples, taken from the chest cavity, were centrifuged $\left(1200 \mathrm{~g}, 10 \mathrm{~min}, 4^{\circ} \mathrm{C}\right.$ ) and plasma stored at $-80^{\circ} \mathrm{C}$ for later biochemical analysis. The right tibia length was measured and the kidneys and epididymal fat pads (from the posterior subcutaneous depots) were weighed. ${ }^{16}$ The hypertrophy index was calculated as the sum of the left and right kidney weights normalized to body weight. Other investigators have previously reported hypertrophy index in the literature when investigating STZ animals, ${ }^{17-19}$ as it is used as a progressive marker of diabetic renal disease.

\section{4 | CINE MRI}

All rodents were imaged on a $7 \mathrm{~T}$ horizontal bore MRI instrument (Varian, Santa Clara, CA, USA), using a $72 \mathrm{~mm}{ }^{1} \mathrm{H} /{ }^{13} \mathrm{C}$ volume transmit coil and a ${ }^{1} \mathrm{H}$ four-channel phased array surface receive coil (RAPID Biomedical, Rimpar, Germany). Eight to ten short-axis (see Figure 2A later) slices (slice thickness, $1.6 \mathrm{~mm}$; matrix size, $128 \times 128 ; T_{\mathrm{E}} / T_{\mathrm{R}}, 4.6 / 1.45 \mathrm{~ms}$; flip angle, 18 ${ }^{\circ}$; number of averages, 4) were acquired with a CINE-FLASH sequence. ${ }^{20}$ Left ventricular volumes at end systole and end diastole were derived using the free-hand drawing function in ImageJ (NIH, Maryland, USA). For each heart, left ventricular mass, ejection fraction, stroke volume and cardiac output were calculated. The average myocardial mass of the left ventricle was obtained from the average of end diastolic and end systolic masses. Stroke volume was obtained from the difference between the end diastolic and end systolic volumes. All structural and functional parameters were also indexed to body weight to account for significant differences in body weight between the control and diabetic animals.

\section{5 | Hyperpolarized MRS}

Experiments were performed between $7 \mathrm{am}$ and $1 \mathrm{pm}$ when rodents were in the fed state. Samples were prepared from $40 \mathrm{mg}$ of either $\left[1-{ }^{13} \mathrm{C}\right]$ pyruvic acid or $\left[2{ }^{13} \mathrm{C}\right]$ pyruvic acid (Sigma), doped with $15 \mathrm{mM}$ trityl radical (OXO63, GE Healthcare, Chicago, USA) and $3 \mu \mathrm{l}$ Dotarem (1:50 dilution, Guerbet, Villepinte, France) and hyperpolarized in a prototype polarizer (Oxford Instruments, Abingdon, UK) operating at a field strength of $3.35 \mathrm{~T}$ and a base temperature of 0.8-1.2 K, with 30-40 min of $100 \mathrm{~mW}$ microwave irradiation at approximately $94 \mathrm{GHz}{ }^{14}$ The sample was subsequently dissolved in a pressurized and heated alkaline solution, containing $2.4 \mathrm{~g} / \mathrm{L}$ sodium hydroxide and $100 \mathrm{mg} / \mathrm{L}$ EDTA (ethylenediaminetetraacetic acid) dipotassium salt (Sigma-Aldrich), to yield a solution of $80 \mathrm{mM}$ hyperpolarized sodium [1- $\left.{ }^{13} \mathrm{C}\right] p y r u v a t e$ or $\left[2-{ }^{13} \mathrm{C}\right]$ pyruvate with a polarization of $30 \%$ or $20 \%$ respectively, at physiological temperature and $\mathrm{pH}$. From the resulting solution, $1 \mathrm{~mL}$ was injected over $10 \mathrm{~s}$ via a tail vein catheter into a rat located in the $7 \mathrm{~T}$ MRI system described above. Using the $72 \mathrm{~mm}{ }^{1} \mathrm{H} /{ }^{13} \mathrm{C}$ volume transmit coil with a $40 \mathrm{~mm}$ two-channel ${ }^{13} \mathrm{C}$ surface receive array with an integrated preamp (RAPID Biomedical), cardiac ${ }^{13} \mathrm{C}$ spectra were acquired using a simple ECG-gated pulse-acquire spectroscopy sequence over $60 \mathrm{~s}$ following the injection of the hyperpolarized pyruvate (repetition time, $1 \mathrm{~s}$; excitation flip angle, 15; sweep width, $13021 \mathrm{~Hz}$; acquired points, 2048; frequency centered on the $\mathrm{C}_{1}$ pyruvate resonance). ${ }^{21}$

Each rat received two injections ( $1 \mathrm{~mL} /$ injection), one with $\left[1-{ }^{13} \mathrm{C}\right]$ pyruvate and one with $\left[2-{ }^{13} \mathrm{C}\right]$ pyruvate, given in a random order and separated by at least $1 \mathrm{~h}$. Following data acquisition, the ${ }^{13} \mathrm{C}$ label from pyruvate and its metabolic products were summed over $30 \mathrm{~s}$ from the first appearance of pyruvate in the acquired spectra and fitted with the AMARES algorithm within jMRUI. ${ }^{22}$ Example summed spectra following injection of $\left[1-{ }^{13} \mathrm{C}\right]$ pyruvate and $\left[2-{ }^{13} \mathrm{C}\right]$ pyruvate are shown later in Figure $3 \mathrm{~A}$ and $3 \mathrm{~B}$, with further example spectra from each of the groups studied shown in Supplementary Figures S1 and S2. Each of the metabolites was quantified as the ratio of the metabolites to either [1- $\left.{ }^{13} \mathrm{C}\right] p y r u v a t e$ or $\left[2-{ }^{13} \mathrm{C}\right]$ pyruvate.

$\mathrm{PDH}$ flux was calculated as the ratio of $\mathrm{CO}_{2}+$ bicarbonate to $\left[1-{ }^{13} \mathrm{C}\right]$ pyruvate measured in the $\left[1-{ }^{13} \mathrm{C}\right]$ pyruvate experiment, a measure that has previously been shown to correlate to PDH activity measured in an ex vivo enzymatic assay. ${ }^{23}$ Subsequently, to assess any changes within the Krebs cycle independent of changes in ${ }^{13} \mathrm{C}$ flux through $\mathrm{PDH}$ (ie the ratio of $\mathrm{CO}_{2}+$ bicarbonate to $\left[1-{ }^{13} \mathrm{C}\right]$ pyruvate), all metabolites obtained from $\left[2-{ }^{13} \mathrm{C}\right]$ pyruvate were normalized to $\mathrm{PDH}$ flux measured in the $\left[1-{ }^{13} \mathrm{C}\right]$ pyruvate experiment conducted in the same animal. 


\section{6 | Langendorff perfusions}

All animals were continued on the treatment protocol for one additional week following MRI/MRS, after which hearts were excised for a Langendorff ischemia/reperfusion protocol. The hearts were cannulated and perfused with warm oxygenated Krebs-Henseleit buffer $\left(37{ }^{\circ} \mathrm{C}\right)$ containing $11 \mathrm{mM}$ glucose and $0.4 \mathrm{mM}$ of palmitate, at a constant pressure of $100 \mathrm{~mm} \mathrm{Hg}$ as described by Heather et al. ${ }^{24}$ A water-filled PVC balloon, which was connected via a polythene tube to a calibrated pressure transducer and a PowerLab data acquisition system (AD Instruments, Oxford, Oxfordshire, UK), was inserted into the left ventricle to measure cardiac function. The balloon was inflated to an end diastolic pressure of 4-8 $\mathrm{mm} \mathrm{Hg}$. Hearts were subjected to $20 \mathrm{~min}$ of normal flow ( $t=1 \mathrm{~min} 20 \mathrm{~s}$ ), followed by $30 \mathrm{~min}$ of a low-flow ischemia ( $0.4 \mathrm{~mL} / \mathrm{min} / \mathrm{gww}$, $t=21 \mathrm{~min} 50 \mathrm{~s}$ ) and reperfused again at normal flow for another $30 \mathrm{~min}(t=51 \mathrm{~min} 80 \mathrm{~s})$. The hearts were freeze-clamped with liquid-nitrogencooled Wallenberger tongs whilst still beating on the perfusion apparatus at $t=80 \mathrm{~min}$. Cardiac function was assessed using the peak systolic pressure, end diastolic pressure, developed pressure (systolic minus end diastolic pressure), heart rate (beats per minute, BPM) and rate pressure product (RPP; developed pressure multiplied by heart rate).

\section{7 | Blood metabolites}

Glucose concentrations were measured from fasted blood samples taken from the tail vein and acquired at one, two and five weeks after STZ injection. Non-esterified fatty acids (NEFAs) were also measured in the fasted blood samples obtained five weeks after STZ/citrate buffer injection using an assay kit (Randox Laboratories, Crumlin, UK), respectively. Terminal fed blood samples were analyzed for 3-hydroxybutyrate (3-OHB), triglycerides (TAGs) and lactic acid using an ABX Pentra 400 (ABX Diagnostics, Kyoto, Japan).

\section{8 | Metabolomics}

Terminal fed blood samples were assessed for low-molecular-weight metabolites with liquid chromatography-mass spectrometry (LC-MS) within the Department of Chemistry, University of Oxford. Plasma samples were filtered through molecular weight cut-off filters (10 kDa) to remove proteins. ${ }^{25}$ The infranatant was recovered and evaporated to dryness under reduced pressure. Sample residue was then resuspended in acetonitrile:water (95\%:5\%). Authenticated standards for selected acylcarnitines (up to $1.0 \mu \mathrm{g} / \mathrm{mL}$ ) were prepared using an identical method.

For LC-MS, acylcarnitines were separated and resolved using hydrophobic-interaction liquid chromatography-mass spectrometry (HILIC). Samples were separated as previously outlined. ${ }^{25,26}$ Briefly, samples were eluted using a binary solvent, acetonitrile:water (50\%:50\%) containing ammonium acetate (10 mM final concentration, Solvent A) and acetonitrile:water (95\%:5\%) containing ammonium acetate (10 mM final concentration, Solvent B). Samples were resolved using a linear gradient (10 min: 100\% Solvent A to 100\% Solvent B) and re-equilibrated with $100 \%$ Solvent A. Putative compounds were identified with reference to authenticated standards for selected acylcarnitines using retention time, accurate mass and fragmentation pattern to identify individual compounds. ${ }^{25}$ Concentrations were calculated with reference to specific standard curves.

\section{9 | Statistics}

All data are presented as mean \pm standard deviation (SD) of the indicated number of rodents (n). Two-way ANOVA was used for assessment of the effect of STZ injection and the effect of meldonium treatment. When an interaction term was significant in the two-way ANOVA, post hoc multiple comparison testing using a Bonferroni correction was used to investigate the effect of meldonium treatment on both the control and diabetic groups. Differences between groups were considered statistically significant if $p<0.05$.

\section{3 | RESULTS}

\section{1 | Animal characterization}

STZ induction in male Wistar rats led to hyperglycemia (>13 mmol/L) observed one week after STZ injection, which gradually increased throughout the course of the experiment (Figure 1A). At five weeks after STZ injection, diabetic animals had markedly elevated glucose levels, which was associated with an elevated hypertrophy index of the kidneys (Figure 1B). Daily injections with meldonium had no significant impact on blood glucose levels or the kidney hypertrophy index. 
(A)

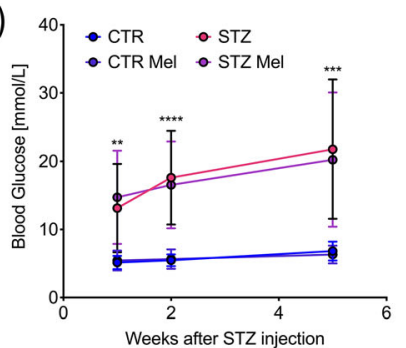

(D)

(G)

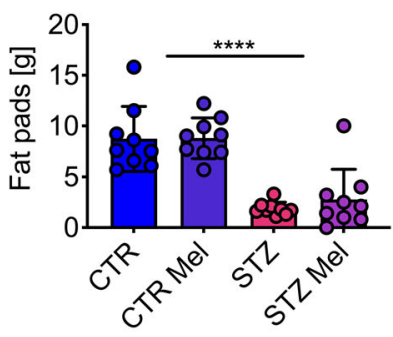

(B)

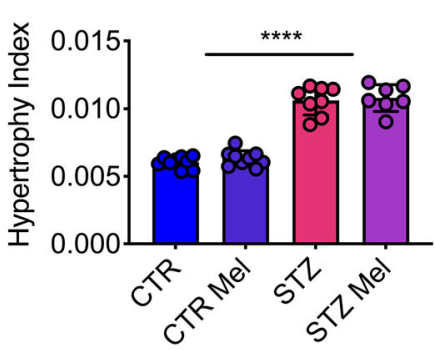

(E)

(H)
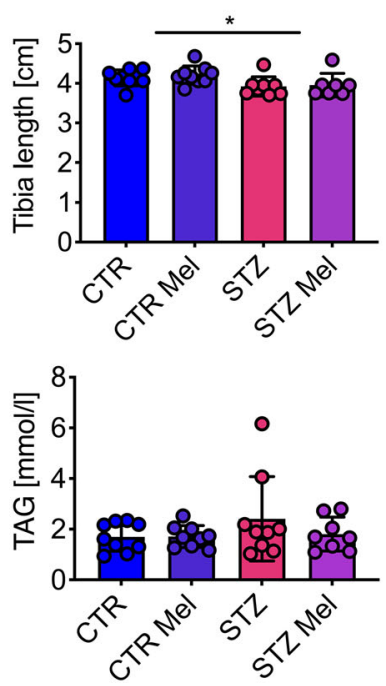

(C)

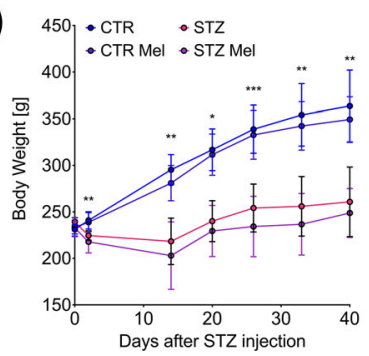

(F)

(I)
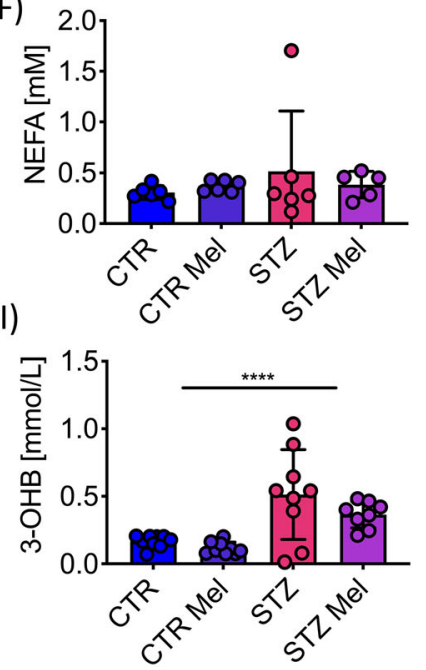

FIGURE 1 Animal characteristics five weeks after STZ-induced diabetes (STZ) compared with citrate buffer injected controls (CTR); half of all animals were treated with meldonium (Mel) initiated at Week 2, generating four different groups. A, Fasted blood glucose concentration at 1,2 and 5 weeks [mmol/L]. B, Hypertrophy index for kidneys; kidney weight normalized to body weight. C, Weight progression over time [g]. D, Epididymal fat pad weights at 5 weeks [g]. E, Tibia length at 5 weeks [cm]. F, Fasted NEFA levels at 5 weeks [mM]. G, Fed cholesterol levels at 5 weeks [mmol/L]. H, Fed TAG concentrations at 5 weeks [mmol/LI]. I, Fed 3-OHB concentrations at 5 weeks [mmol/l]. Data presented as mean $\pm \mathrm{SD} .{ }^{*} p<0.05,{ }^{* *} p<0.01,{ }^{* * *} p<0.001,{ }^{* * * *} p<0.0001$

Diabetic animals failed to gain weight over the course of the study, leading to a significant difference in body weight between controls and diabetics at all time-points after the initial weight matching (Figure 1C). Lack of weight gain in the diabetic animals was attributed primarily to a reduction in fat mass as indicated by a 4.7-fold reduction in epididymal fat pad weight (Figure 1D). There was also a small, but significant, reduction in lean mass as measured by a decreased tibia length at the terminal time-point in the diabetic animals (Figure 1E). No differences in NEFA, TAG or cholesterol concentrations were observed in the diabetic animals; however, STZ injection resulted in a significant elevation in the levels of the ketone body, 3-OHB (Figure 1F-I). Meldonium treatment had no significant effect on any of the plasma metabolites assessed.

\section{2 | Cardiac function}

Myocardial mass was reduced by $28 \%$ in the diabetic animals compared to the control animals (Figure $2 \mathrm{~B}$ ), but this was in proportion to the change in body weight, with no significant difference observed in the heart weight to body weight ratio (Table 1). End diastolic volume in the diabetic animals was reduced by $16 \%$, leading to an $18 \%$ reduction in stroke volume and, when combined with a significantly decreased heart rate, a $29 \%$ lower cardiac output in comparison with the control animals (Figure 2C-F). When differences in body weight were accounted for, this meant that a significant increase in stroke index was observed in the diabetic animals, which balanced the decrease in heart rate and led to no significant change in cardiac index (Table 1). Taken together with the lack of change in ejection fraction (Figure 2G), these structural and functional characterizations demonstrate that the diabetic hearts had no overt changes in systolic function apart from those induced by the reduction in body weight. Meldonium had no significant effect on any of the cardiac structural or functional parameters assessed.

\section{3 | Cardiac metabolism}

As has been previously observed, ${ }^{27} \mathrm{PDH}$ flux, as assessed by bicarbonate and $\mathrm{CO}_{2}$ production from the injected hyperpolarized $\left[1-{ }^{13} \mathrm{C}\right]$ pyruvate, was significantly reduced in the diabetic heart. In addition, meldonium treatment led to a significant increase in PDH flux, by approximately 
(A)
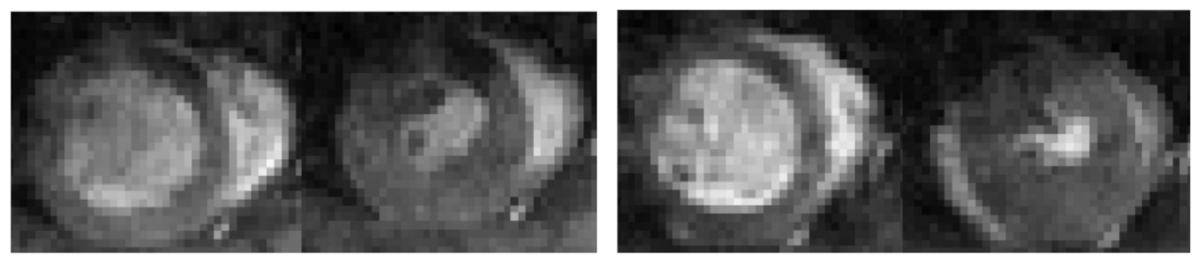

(B)

(E)

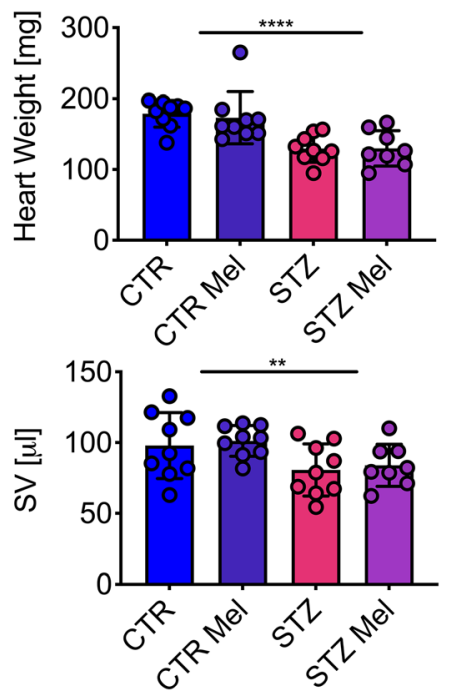

(C) 200

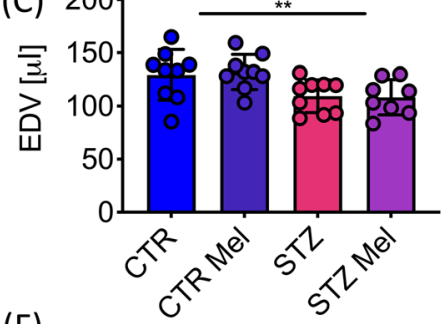

(F)

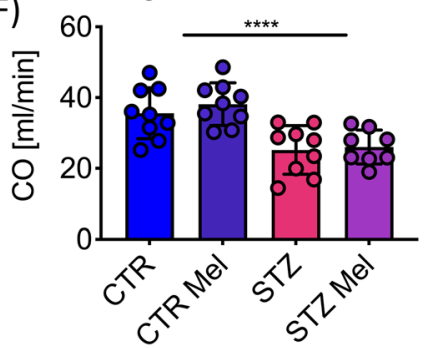

(D) 80

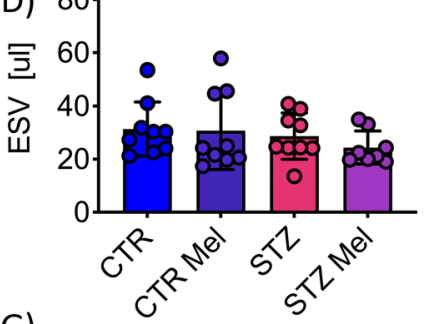

(G)

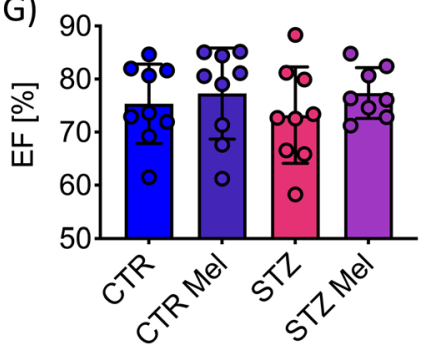

FIGURE 2 In vivo effects on cardiac function. A, Example CINE MRI images from diastole to systole, short-axis view. B, Average myocardial wall mass $[\mathrm{mg}]$. C, End diastolic volume (EDV) $[\mu \mathrm{L}]$. D, End systolic volume (ESV) $[\mu \mathrm{L}]$. E, Stroke volume (SV) $[\mu \mathrm{L}] . \mathrm{F}, \mathrm{Cardiac}$ output $(\mathrm{CO})[\mathrm{mL} / \mathrm{min}]$. (G) Ejection fraction (EF) [\%]. Data presented as mean \pm SD. ${ }^{* *} p<0.01,{ }^{* * *} p<0.0001$

TAB LE 1 Cardiac function measured using CINE MRI. The table shows functional parameters, where most are normalized to body weight (BW). Data presented as mean \pm SD. Two-way ANOVA was undertaken and the effects of an interaction, STZ and meldonium are displayed with $p$-values, where $p<0.05$ was considered significant

\begin{tabular}{|c|c|c|c|c|c|c|c|}
\hline & \multicolumn{2}{|l|}{ Control } & \multicolumn{2}{|l|}{ STZ } & $\begin{array}{l}\mathrm{STZ} \\
p\end{array}$ & $\begin{array}{l}\text { Meldonium } \\
p\end{array}$ & $\begin{array}{l}\text { Interaction } \\
p\end{array}$ \\
\hline $\mathrm{HW} / \mathrm{BW}$ & $0.56 \pm 0.13$ & $0.48 \pm 0.07$ & $0.54 \pm 0.05$ & $0.55 \pm 0.06$ & 0.46 & 0.17 & 0.12 \\
\hline Heart rate & $370 \pm 32$ & $380 \pm 32$ & $310 \pm 31$ & $310 \pm 32$ & $<0.0001$ & 0.59 & 0.70 \\
\hline End systolic volume/BW & $0.098 \pm 0.031$ & $0.085 \pm 0.037$ & $0.12 \pm 0.04$ & $0.10 \pm 0.022$ & 0.072 & 0.18 & 0.82 \\
\hline End diastolic volume/BW & $0.40 \pm 0.09$ & $0.37 \pm 0.02$ & $0.46 \pm 0.05$ & $0.46 \pm 0.07$ & 0.0012 & 0.42 & 0.35 \\
\hline Cardiac output/BW (cardiac index) & $0.11 \pm 0.028$ & $0.11 \pm 0.014$ & $0.10 \pm 0.019$ & $0.11 \pm 0.014$ & 0.75 & 0.93 & 0.41 \\
\hline
\end{tabular}

Abbreviation: HW, Heart weight.

threefold in the diabetic animals and 1.2-fold in the control animals (Figure 3C). Neither STZ injection nor meldonium treatment had any effect on the incorporation of the ${ }^{13} \mathrm{C}$ label from $\left[1-{ }^{13} \mathrm{C}\right]$ pyruvate into either lactate or alanine (Figure $3 \mathrm{D}$ and $3 \mathrm{E}$ ).

After normalization for the differences observed in $\mathrm{PDH}$ flux, and thus transfer of the ${ }^{13} \mathrm{C}$ label into the TCA cycle, no significant differences in ${ }^{13} \mathrm{C}$ label incorporation into citrate or glutamate were observed in any of the groups (Figure $3 \mathrm{~F}-\mathrm{H}$ ). However, meldonium treatment led to a 2.1-fold reduction in the incorporation of the ${ }^{13} \mathrm{C}$ label into acetylcarnitine in both control and diabetic animals (Figure $\left.3 \mathrm{H}\right)$, indicating a reduced availability of L-carnitine in the meldonium treated heart.

\section{4 | Plasma metabolomics}

As expected, meldonium treatment led to a significant elevation in the plasma levels of meldonium and a significant reduction in plasma L-carnitine in both control and diabetic animals. This reduction was observed in addition to a significant reduction in L-carnitine levels due to STZ-induced 
(A)

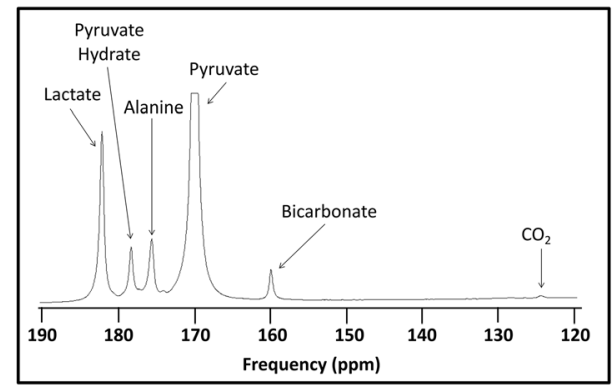

(C)

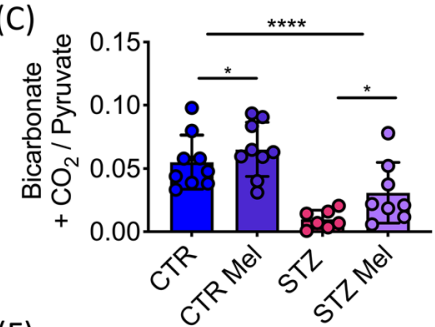

(F)

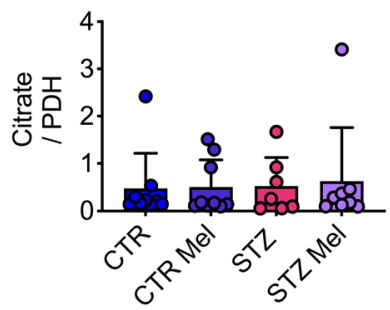

(D)

(G)
(B)
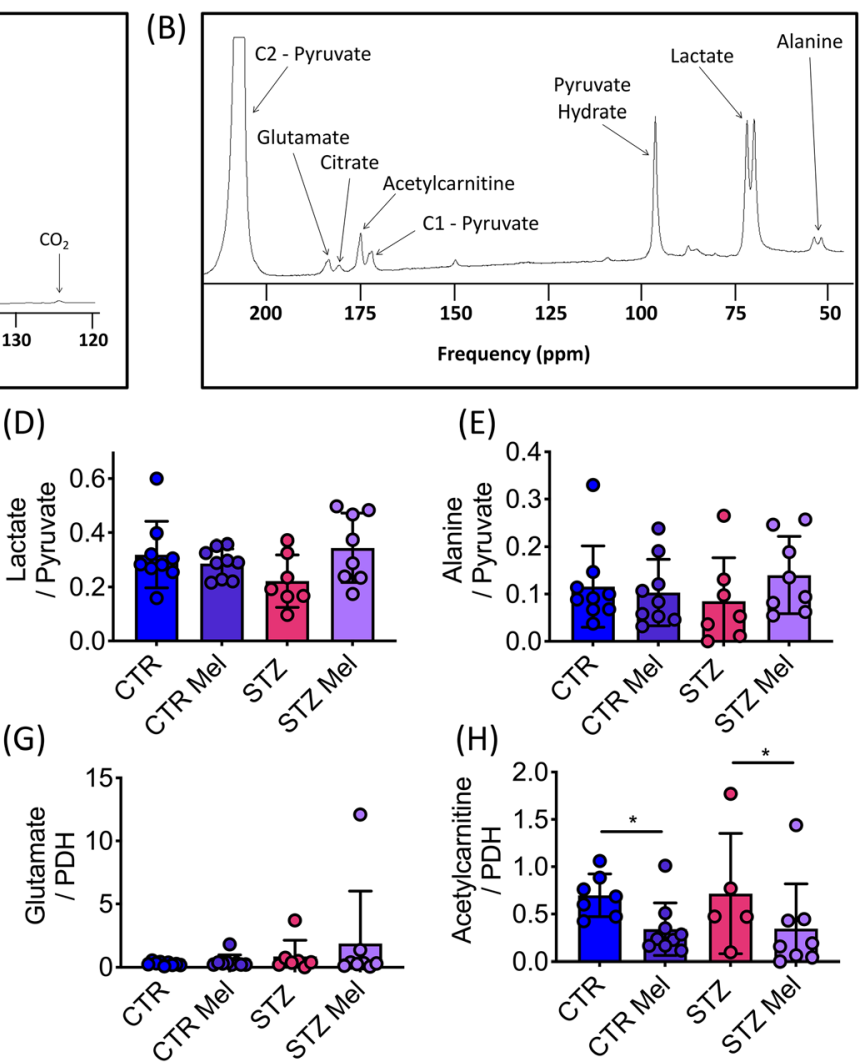

(E)

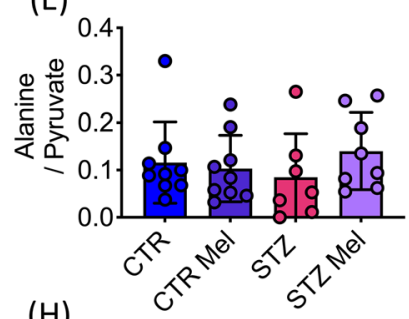

(H)

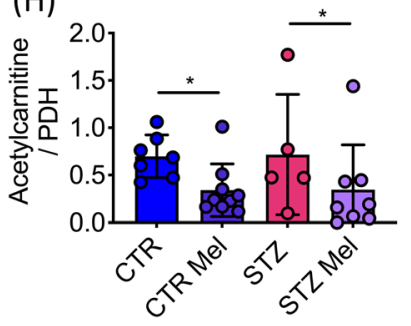

FI GURE 3 In vivo effects on cardiac metabolism. A, Example of a $30 \mathrm{~s}$ summed $\left[1{ }^{13} \mathrm{C}\right]$ pyruvate spectrum. B, Example of a $30 \mathrm{~s}$ summed $\left[2-{ }^{13} \mathrm{C}\right]$ pyruvate spectrum. C, Bicarbonate $+\mathrm{CO}_{2} /$ pyruvate ratio, a marker of $\mathrm{PDH}$ flux. D, Lactate/pyruvate ratio. E, Alanine/pyruvate ratio. $\mathrm{F}$, Citrate/pyruvate ratio normalized to $\mathrm{PDH}$-flux. G, Glutamate/pyruvate ratio normalized to $\mathrm{PDH}$-flux. $\mathrm{H}$, Acetylcarnitine/pyruvate ratio normalized to PDH-flux. Data presented as mean $\pm \mathrm{SD} .{ }^{*} p<0.05,{ }^{* * * *} p<0.0001$

diabetes (Figure 4A and 4B). Similar patterns to those seen in L-carnitine levels were also observed with short-chain (C3) and long-chain (C14/C18) acylcarnitine species (Figure 4C-F). Plasma levels of all acylcarnitine species assessed are presented in Table 2.

\section{5 | Post-ischemic recovery}

As a marker of cardiac function, RPP was 31\% lower in the diabetic animals before ischemia and this functional impairment worsened after ischemia with RPP 55\% lower in the diabetic hearts (Figure 5A-C). The observed impairment in post-ischemic function in the diabetic heart was due to reductions in both systolic pressure (55\% reduction) and heart rate (13\% reduction) when compared with the control animals (Figure 5D and 5E).

However, meldonium treatment led to a significant elevation in post-ischemic RPP by 1.3-fold in the control animals and 1.5-fold in the diabetic animals (Figure 5C). This improvement in post-ischemic function was driven by small (but non-significant) increases in both developed pressure and heart rate in the controls (Table 3 ), whilst it was driven by a significant $70 \%$ elevation in systolic pressure in the diabetic hearts (Figure 5D).

\section{4 | DISCUSSION}

\subsection{Overview}

Diabetic patients are more likely to develop ischemic heart disease and suffer increased mortality and morbidity following a myocardial infarction. ${ }^{28}$ Whilst the pathophysiology of this increased burden of disease is complex and multi-factorial, alterations in cardiac metabolism, with a shift towards reduced glucose metabolism, are considered to play a significant role. Therefore, therapeutic interventions that target elevation of glucose metabolism may offer some benefit to the diabetic population. As an example, meldonium has been shown to be an effective anti-ischemic drug, which is proposed to switch the balance of fuel utilization away from fatty acid oxidation and towards glucose oxidation. Meldonium has 
(A)
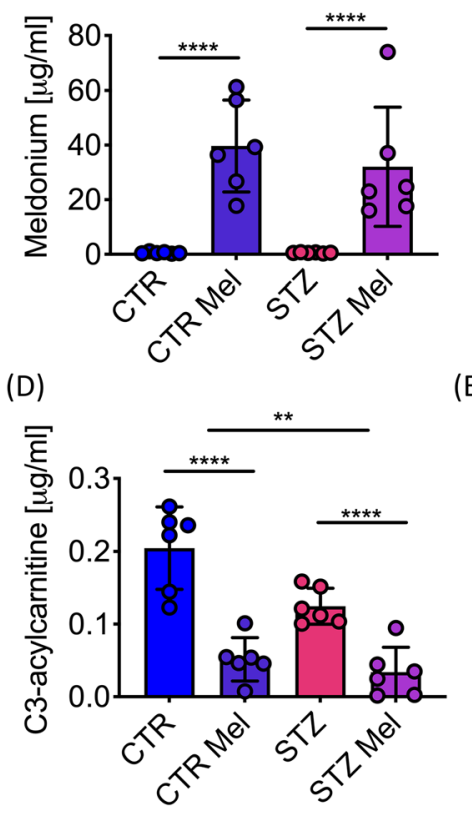

(D)
(B)
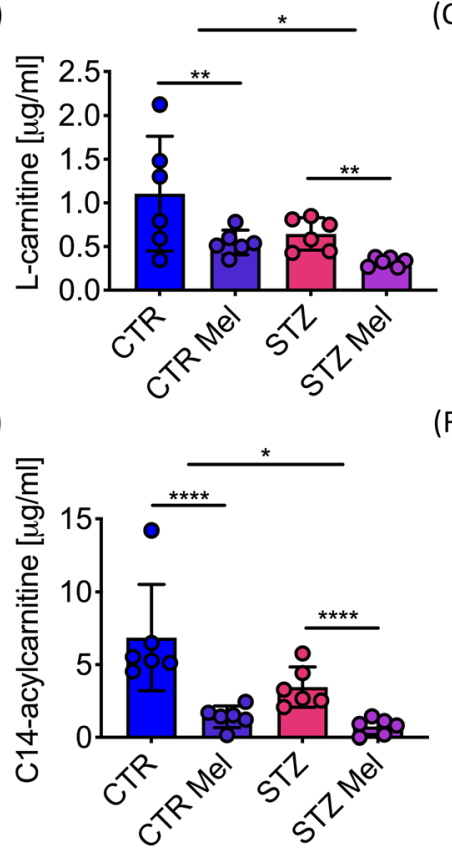

(C)

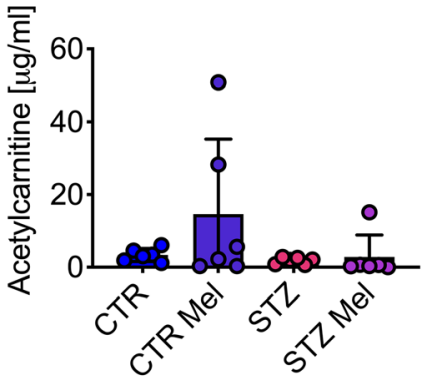

(F)

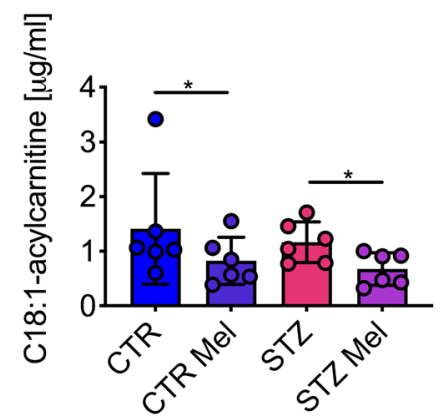

FIGURE 4 A, Meldonium concentration levels in plasma $[\mu \mathrm{g} / \mathrm{mL}]$. B, Free L-carnitine concentration in plasma $[\mu \mathrm{g} / \mathrm{mL}]$. C, Acetylcarnitine concentration levels in plasma $[\mu \mathrm{g} / \mathrm{mL}]$. D, C3-acylcarnitine (propionylcarnitine) concentration levels in plasma $[\mu \mathrm{g} / \mathrm{mL}]$. E, C14-acylcarnitine (tetradecanoylcarnitine) concentration levels in plasma $[\mu \mathrm{g} / \mathrm{mL}]$. F, C18:1-acylcarnitine (stearoylcarnitine) concentration levels in plasma $[\mu \mathrm{g} / \mathrm{mL}]$. Data presented as mean \pm SD. ${ }^{*} p<0.05,{ }^{* *} p<0.01,{ }^{* * * *} p<0.0001$

TAB LE 2 Metabolomics were undertaken in the plasma of the animals. Different lengths of acylcarnitine species are displayed. Data presented as mean \pm SD. Two-way ANOVA undertaken and the effects of an interaction, STZ and meldonium are displayed with $p$-values, where $p<0.05$ was considered significant

\begin{tabular}{|c|c|c|c|c|c|c|c|}
\hline Metabolites [ $\mu \mathrm{g} / \mathrm{ml}]$ & \multicolumn{2}{|l|}{ Control } & \multicolumn{2}{|l|}{ STZ } & $\begin{array}{l}\text { STZ } \\
p\end{array}$ & $\begin{array}{l}\text { Meldonium } \\
p\end{array}$ & $\begin{array}{l}\text { Interaction } \\
p\end{array}$ \\
\hline Meldonium & $0.54 \pm 0.33$ & $40 \pm 0.17$ & $0.55 \pm 0.17$ & $32 \pm 22$ & 0.51 & $<0.0001$ & 0.51 \\
\hline L-carnitine & $1.11 \pm 0.66$ & $0.55 \pm 0.14$ & $0.65 \pm 0.19$ & $0.32 \pm 0.05$ & 0.026 & 0.0059 & 0.41 \\
\hline Acetylcarnitine & $3.4 \pm 1.8$ & $15 \pm 21$ & $1.8 \pm 0.090$ & $2.8 \pm 6.0$ & 0.15 & 0.18 & 0.26 \\
\hline C3-acylcarnitine & $0.20 \pm 0.057$ & $0.052 \pm 0.030$ & $0.12 \pm 0.025$ & $0.034 \pm 0.034$ & 0.0055 & $<0.0001$ & 0.061 \\
\hline C6-acylcarnitine & $12 \pm 2.7$ & $3.6 \pm 1.8$ & $5.2 \pm 1.4$ & $2.6 \pm 2.1$ & 0.0002 & $<0.0001$ & 0.0027 \\
\hline C14-acylcarnitine & $6.9 \pm 3.7$ & $1.4 \pm 0.74$ & $3.5 \pm 1.4$ & $0.75 \pm 0.55$ & 0.022 & $<0.0001$ & 0.11 \\
\hline C18-acylcarnitine & $2.8 \pm 2.5$ & $1.0 \pm 0.45$ & $1.7 \pm 0.64$ & $1.0 \pm 0.75$ & 0.32 & 0.035 & 0.35 \\
\hline C18:1-acylcarnitine & $1.4 \pm 1.02$ & $0.82 \pm 0.43$ & $1.7 \pm 0.37$ & $0.67 \pm 0.30$ & 0.43 & 0.040 & 0.85 \\
\hline
\end{tabular}

been shown to work by lowering myocardial free L-carnitine and long-chain acylcarnitine by more than $60 \%$. This is proposed to lead to a suppression of free fatty acid oxidation, which should lead to a reciprocal increase in glucose oxidation and may account for the observed myocardial protection during ischemia. ${ }^{29}$

\section{\begin{tabular}{l|l}
4.2 & Diabetes
\end{tabular}}

This study has shown that, as expected, STZ-induced type 1 diabetic rats had hyperglycemia accompanied by an elevated hypertrophy index in the kidneys. These are common features of STZ-induced diabetes, with elevated glucose levels already known to be apparent after $2 \mathrm{~d} .^{30}$ The elevated hypertrophy index is mostly accounted for by increased fluid intake, as shown by Bauman, ${ }^{31}$ with an element of increased renal mass considered to account for the rest. Furthermore, the STZ-injected animals had reduced body weight accompanied by both significantly smaller fat 
(A)

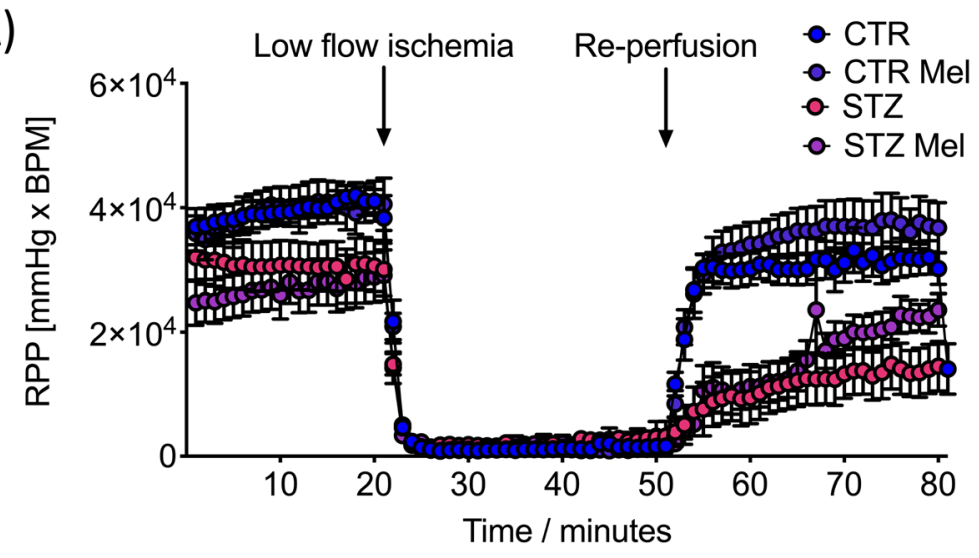

(B)

(D)

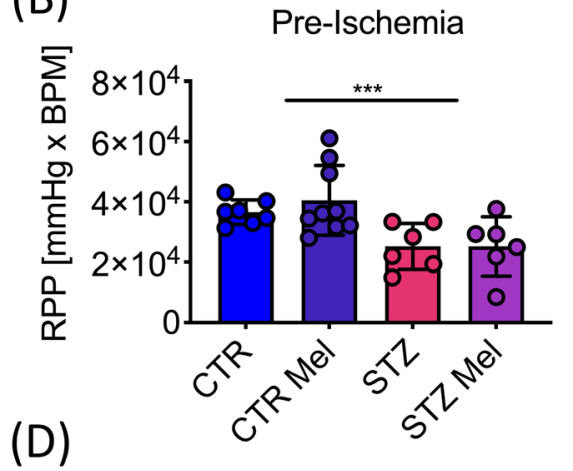

(C)

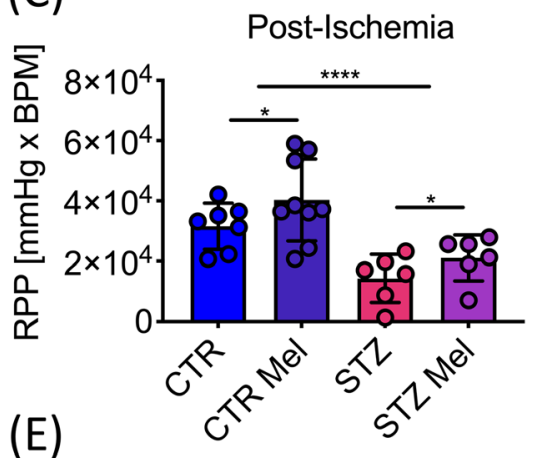

Post-Ischemia

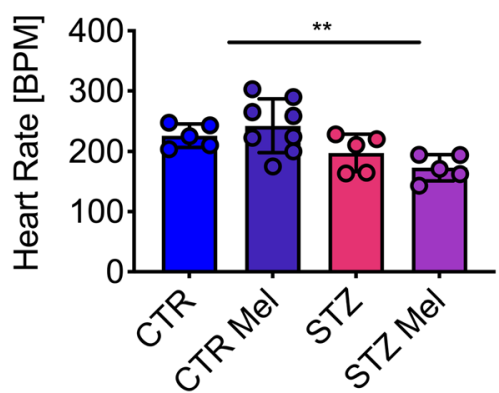

FIGURE 5 A, RPP over time [mm Hg $\times$ BPM]. Before ischemia ( $t=1 \mathrm{~min} 20 \mathrm{~s}$ ), low-flow ischemia ( $t=21 \mathrm{~min} 50 \mathrm{~s})$, after ischemia $(t=52 \mathrm{~min}$ $80 \mathrm{~s}) . \mathrm{B}, \mathrm{RPP}$ before ischemia [mm Hg $\times$ BPM]. C, RPP after ischemia [mm Hg $\times$ BPM]. D, Developed pressure after ischemia [mm Hg]. E, Heart rate after ischemia [BPM]. Data presented as mean \pm SD. ${ }^{*} p<0.05,{ }^{* *} p<0.01,{ }^{* * *} p<0.001,{ }^{* * * *} p<0.0001$

pads and reduced tibia length. Cardiac structure (left ventricle mass) and function (stroke volume, cardiac output) were reduced in the STZinjected animals relative to controls, but these changes were proportionate to the reduction in body weight. When indexed to body weight, a slight increase in stroke index was observed to balance the significant reduction in heart rate, allowing the diabetic animals to maintain a normal cardiac index. As the CINE MRI techniques used in this study only allow assessment of systolic function, these findings do not exclude the possibility that there may have been an element of diastolic dysfunction, as has previously been seen in diabetic animal models when assessed using echocardiography. ${ }^{13}$ in vivo metabolism, assessed with hyperpolarized magnetic resonance, showed reduced PDH flux in the STZ injected animals, which has been shown previously five days after STZ induction. ${ }^{27}$

\section{3 | Meldonium treatment in diabetic animals}

Meldonium treatment had minimal effects on physical and plasma metabolite parameters assessed in the STZ-injected animals (eg blood glucose, body weight, hypertrophy index etc). Previous studies have shown that meldonium can reduce blood glucose levels in both rodent models of 
TAB LE 3 A Langendorff ischemia/reperfusion protocol was undertaken. The functional parameters obtained before ischemia, during ischemia and after ischemia are displayed. Data presented as mean \pm SD. Two-way ANOVA was undertaken and the effects of an interaction, STZ and meldonium (Mel) are displayed with $p$-values, where $p<0.05$ was considered significant

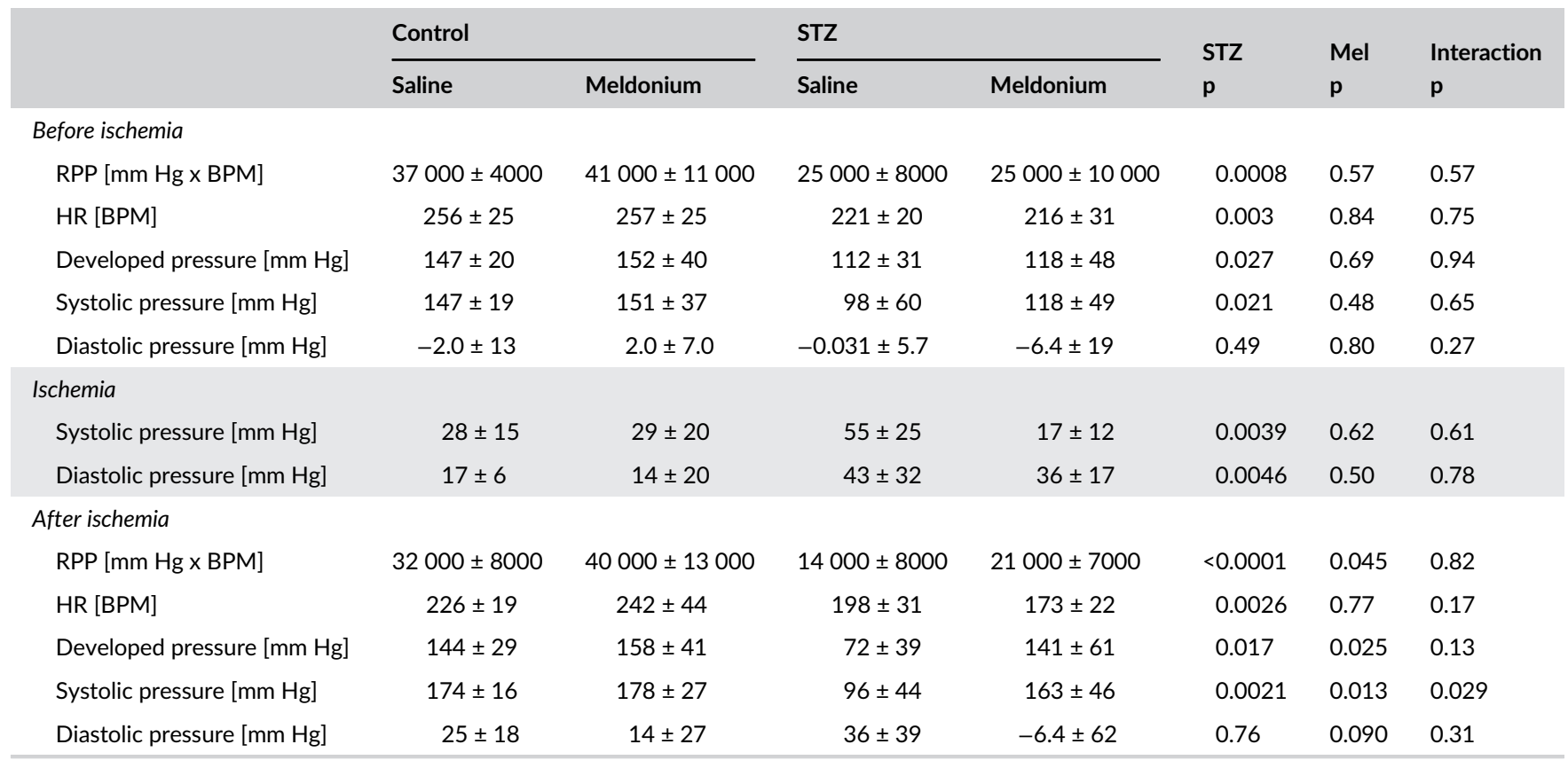

diabetes (STZ injection) and insulin-resistant models of diabetes (Zucker obese rats), as well as in humans with type 2 diabetes. ${ }^{32-34}$ However, treatment in those studies was continued for longer periods (4-12 weeks) than in our current study ( 3 weeks) and so it is possible that a longer period of treatment might have revealed these beneficial effects.

However, we did observe that meldonium induced an increase in flux through $\mathrm{PDH}$, as indicated by enhanced production of ${ }^{13} \mathrm{C}$ bicarbonate and ${ }^{13} \mathrm{CO}_{2}$ following the injection of hyperpolarized $\left[1-{ }^{13} \mathrm{C}\right]$ pyruvate. In addition, plasma metabolomics and the use of hyperpolarized $\left[2-{ }^{13} \mathrm{C}\right]$ pyruvate revealed a reduction in L-carnitine availability, leading to reduced incorporation of the ${ }^{13} \mathrm{C}$ label into acetylcarnitine. Taken together, this suggests an increase in glucose oxidation in the meldonium-treated diabetic heart and a possible shift away from fatty acid oxidation. However, the exact effect of meldonium on fatty acid oxidation is unclear, with a previous study showing the potential for increased fatty acid oxidation in the mitochondria of meldonium-treated hearts. ${ }^{35}$ Unfortunately, we were unable to directly assess fatty acid oxidation in vivo as hyperpolarized MRI is currently unable to probe the oxidation of long-chain fatty acids, although development work has shown the ability of the technique to probe the oxidation of short-chain fatty acids and ketone bodies. ${ }^{36-42}$ Fatty acid oxidation can be assessed in the ex vivo perfused heart using either radiolabeled or ${ }^{13} \mathrm{C}$-labeled fatty acids. However, such experiments are less physiological than experiments performed in vivo, as the results can be affected by the concentrations of different substrates (eg glucose, fatty acids) contained within the perfusion buffer and the lack of circulating hormones (eg insulin) seen in vivo.

Improvements in post-ischemic function were observed in the ex vivo perfused heart with a 50\% increase in RPP following a 30 min period of low-flow ischemia. This result agrees with the work of Vilskersts et $\mathrm{al}^{43}$ and Sesti et $\mathrm{al}^{44}$ who have shown that meldonium treatment leads to a smaller infarct size after ischemia/reperfusion. Meldonium treatment has also previously been shown to improve diastolic heart function and increase left ventricular ejection fraction in patients with type 2 diabetes, with blood glucose levels also reduced in these patients. ${ }^{45}$ The mechanistic link between these improvements and meldonium's effect on L-carnitine availability has been demonstrated by simultaneous treatment with meldonium and L-carnitine, which prevents these beneficial effects. ${ }^{46}$

\subsection{Meldonium treatment in control animals}

Meldonium treatment induced very few effects in the healthy control animals but it increased PDH flux by $20 \%$, likely indicating increased glucose oxidation. The mechanism for this increase would again appear to be mediated by a reduction in fatty acid oxidation due to the reduced availability of L-carnitine for transport of fatty acids into the mitochondria. In support of this, hyperpolarized MRS also revealed a reduction in mitochondrial L-carnitine availability in the control animals, as demonstrated by a reduction in the incorporation of $\left[2-{ }^{13} \mathrm{C}\right]$ pyruvate into acetylcarnitine, a finding supported by significant reductions in several acylcarnitine species in the plasma, including free L-carnitine. 
Meldonium showed no differences in ex vivo cardiac function in the control hearts prior to ischemia; however, after ischemia RPP was elevated, suggesting an improved recovery. Such a finding indicates that, even in the control heart, a switch towards increased flux through $\mathrm{PDH}$ is beneficial during reperfusion. A similar finding has previously been shown by Liu et al when using the PDH activator dichloroacetate. ${ }^{47}$

\section{5 | Study limitations}

First, the in vivo metabolic data in this study were acquired using cardiac localization achieved through a surface receive coil placed directly over the heart. Previous imaging studies have demonstrated that the bicarbonate signal (and therefore all metabolic products downstream of $\mathrm{PDH}$ ) only originates from the heart ${ }^{48}$; however, there is a possibility that the recorded lactate resonance may include some contamination from the nearby liver and/or lactate that has washed into the sensitive region of the coil in the blood. ${ }^{49}$ Whilst this means that there is no impact on the key findings in this paper (namely increased in PDH flux and reduced incorporation of PDH derived acetyl-CoA into acetylcarnitine in the meldonium-treated heart), to conclusively prove that meldonium does not have an impact on the incorporation of the ${ }^{13} \mathrm{C}$ label from $\left[1-{ }^{13} \mathrm{C}\right]$ pyruvate into $\left[1-{ }^{13} \mathrm{C}\right]$ lactate would require imaging studies to better localize the derived signals to the myocardial tissue.

Second, the concentration of pyruvate injected $(80 \mathrm{mM})$ is supraphysiological and so there is the potential that the pyruvate may have a physiological impact. However, the actual dose of pyruvate delivered is relatively small $(80 \mu \mathrm{mol})$ and is rapidly distributed, diluted and metabolized. Previously published work has measured the circulating concentration of pyruvate following the hyperpolarized injection and shown it to peak at $250 \mu \mathrm{M} 1 \mathrm{~min}$ after the injection. Such a concentration is only four times higher than the baseline plasma pyruvate concentration of $60 \mu \mathrm{M}$ and is in the physiological range of pyruvate concentrations reached during exercise. ${ }^{23}$

Finally, we choose to undertake the $\left[1-{ }^{13} \mathrm{C}\right]$ pyruvate and $\left[2-{ }^{13} \mathrm{C}\right]$ pyruvate experiments separately with the two injections separated by at least $1 \mathrm{~h}$. These experiments could have been performed together using either a co-polarized mixture of the individually labeled pyruvic acid preparations or using dual-labeled $\left[1,2-{ }^{13} \mathrm{C}\right]$ pyruvic acid, which would have reduced the number of injections that needed to be carried out. ${ }^{50} \mathrm{However}$, with such approaches, the $\left[5-{ }^{13} \mathrm{C}\right]$ glutamate and $\left[1-{ }^{13} \mathrm{C}\right]$ acetylcarnitine resonances overlap to some extent with the $\left[1-{ }^{13} \mathrm{C}\right]$ lactate and $\left[1-{ }^{13} \mathrm{C}\right]$ pyruvate peaks, respectively, making them difficult to measure when $B_{0}$ homogeneity is poor. We therefore chose to undertake these as separate experiments to improve the clarity of the acquired spectra.

\section{5 | CONCLUSION}

Therapeutic interventions aimed at restoring the normal balance of glucose and fatty acid oxidation in the diabetic heart have been suggested to have potential in preventing the increased cardiovascular mortality and morbidity associated with the disease. In this study, we have used the novel imaging approach of hyperpolarized magnetic resonance to show that the anti-ischemic agent meldonium leads to an increase in in vivo flux through PDH in both the healthy and diabetic rodent heart. This potentially results in a switch in fuel utilization towards more oxygen-efficient glucose metabolism and may account for the improved recovery after ischemia. Given the recent demonstration of the ability of hyperpolarized magnetic resonance to study alterations in metabolism in the human heart, ${ }^{51,52}$ there is clear potential for such studies to be translated into clinical trials assessing the potential for metabolic therapies in the diabetic heart.

\section{ACKNOWLEDGEMENTS}

Metabolomic analysis was performed in the Department of Chemistry, University of Oxford, by DH led by Associate Professor James McCullagh. This work was supported by the Danish Council for Strategic Research entitled "LIFE-DNP: hyperpolarized magnetic resonance for in vivo quantification of lipid, sugar and amino acid metabolism in lifestyle related diseases". DS would like to acknowledge the financial support provided by a Novo Nordisk Postdoctoral Fellowship. LH acknowledges a BHF Intermediate Research Fellowship (FS/17/58/33072). DJT acknowledges the support provided by the OXFORD-BHF Centre for Research Excellence (grant RE/13/1/30181), the NIHR Oxford Biomedical Research Centre and a BHF Senior Research Fellowship (FS/17/58/33072).

\section{CONFLICT OF INTEREST}

No competing interest declared.

\section{DATA AVAILABILITY STATEMENT}

The data that support the findings of this study are available on request from the corresponding author. The data are not publicly available due to privacy or ethical restrictions. 


\section{ORCID}

Dragana Savic (D) https://orcid.org/0000-0002-2181-3196

Damian J. Tyler (D) https://orcid.org/0000-0002-0780-8905

\section{REFERENCES}

1. Saeedi P, Petersohn I, Salpea P, et al. Global and regional diabetes prevalence estimates for 2019 and projections for 2030 and 2045 : results from the International Diabetes Federation Diabetes Atlas, 9th edition. Diabetes Res Clin Pract. 2019;157:107843. https://doi.org/10.1016/J.DIABRES.2019. 107843

2. International Diabetes Federation. IDF Diabetes Atlas. 9th ed. Brussels, Belgium: International Diabetes Federation; 2019.

3. Kannel WB, McGee DL. Diabetes and cardiovascular disease. J Am Med Assoc. 1979;241(19):2035-2038. https://doi.org/10.1001/jama.1979. 03290450033020

4. Jia G, Whaley-Connell A, Sowers JR. Diabetic cardiomyopathy: a hyperglycaemia- and insulin-resistance-induced heart disease. Diabetologia. 2018;61 (1):21-28. https://doi.org/10.1007/s00125-017-4390-4

5. Soedamah-Muthu SS, Fuller JH, Mulnier HE, Raleigh VS, Lawrenson RA, Colhoun HM. All-cause mortality rates in patients with type 1 diabetes mellitus compared with a non-diabetic population from the UK general practice research database, 1992-1999. Diabetologia. 2006;49(4):660-666. https://doi.org/10.1007/s00125-005-0120-4

6. Isfort M, Stevens SCW, Schaffer S, Jong CJ, Wold LE. Metabolic dysfunction in diabetic cardiomyopathy. Heart Fail Rev. 2014;19(1):35-48. https://doi. org/10.1007/s10741-013-9377-8

7. Scheuermann-Freestone M, Madsen PL, Manners D, et al. Abnormal cardiac and skeletal muscle energy metabolism in patients with type 2 diabetes. Circulation. 2003;107(24):3040-3046. https://doi.org/10.1161/01.CIR.0000072789.89096.10

8. Doenst T, Nguyen TD, Abel ED. Cardiac metabolism in heart failure: implications beyond ATP production. Circ Res. 2013;113(6):709-724. https://doi. org/10.1161/CIRCRESAHA.113.300376

9. Ballard F, Nagele S, Bing RJDW. Myocardial metabolism of fatty acids. J Clin Invest. 1960;39(5):717-723.

10. Randle PJ, Garland PB, Hales CN, Newsholme EA. The glucose fatty-acid cycle its role in insulin sensitivity and the metabolic disturbances of diabetes mellitus. Lancet. 1963;281(7285):785-789. https://doi.org/10.1016/S0140-6736(63)91500-9

11. Hue L, Taegtmeyer H. The Randle cycle revisited: a new head for an old hat. Am J Physiol Endocrinol Metab. 2009;297(3):E578-E591. https://doi.org/ 10.1152/ajpendo.00093.2009

12. Park S, Jeon JH, Min BK, et al. Role of the pyruvate dehydrogenase complex in metabolic remodeling: differential pyruvate dehydrogenase complex functions in metabolism. Diabetes Metab J. 2018;42(4):270-281. https://doi.org/10.4093/dmj.2018.0101

13. Le Page LM, Rider OJ, Lewis AJ, et al. Increasing pyruvate dehydrogenase flux as a treatment for diabetic cardiomyopathy: a combined ${ }^{13} \mathrm{C}$ hyperpolarized magnetic resonance and echocardiography study. Diabetes. 2015;64(8):2735-2743. https://doi.org/10.2337/db14-1560

14. Ardenkjaer-Larsen JH, Fridlund B, Gram A, et al. Increase in signal-to-noise ratio of $>10,000$ times in liquid-state NMR. Proc Natl Acad Sci U S A. 2003;100(18):10158-10163. https://doi.org/10.1073/pnas.1733835100

15. Timm KN, Miller JJ, Henry JA, Tyler DJ. Cardiac applications of hyperpolarised magnetic resonance. Prog Nucl Magn Reson Spectrosc. 2018;106-107: 66-87. https://doi.org/10.1016/j.pnmrs.2018.05.002

16. Chusyd DE, Wang D, Huffman DM, Nagy TR. Relationships between rodent white adipose fat pads and human white adipose fat depots. Front Nutr. 2016;3:10. https://doi.org/10.3389/fnut.2016.00010

17. Habibuddin M, Daghriri HA, Humaira T, Al Qahtani MS, Hefzi AAH. Antidiabetic effect of alcoholic extract of Caralluma sinaica L. on streptozotocininduced diabetic rabbits. J Ethnopharmacol. 2008;117(2):215-220. https://doi.org/10.1016/j.jep.2008.01.021

18. Malatiali S, Francis I, Barac-Nieto M. Phlorizin prevents glomerular hyperfiltration but not hypertrophy in diabetic rats. Exp Diabetes Res. 2008;2008: 305403. https://doi.org/10.1155/2008/305403

19. Lee S-I, Kim J-S, Oh S-H, Park K-Y, Lee H-G, Kim S-D. Antihyperglycemic effect of Fomitopsis pinicola extracts in streptozotocin-induced diabetic rats. J Med Food. 2008;11(3):518-524. https://doi.org/10.1089/jmf.2007.0155

20. Haase A, Frahm J, Matthaei D, Hanicke W, Merboldt K-D. FLASH imaging. Rapid NMR imaging using low flip-angle pulses. J Magn Reson. 1986;67(2): 258-266. https://doi.org/10.1016/0022-2364(86)90433-6

21. Dodd MS, Ball V, Bray R, et al. In vivo mouse cardiac hyperpolarized magnetic resonance spectroscopy. J Cardiovasc Magn Reson. 2013;15:19. https:// doi.org/10.1186/1532-429X-15-19

22. Vanhamme L, Van Den Boogaart A, Van Huffel S. Improved method for accurate and efficient quantification of MRS data with use of prior knowledge. J Magn Reson. 1997;129(1):35-43. https://doi.org/10.1006/jmre.1997.1244

23. Atherton $\mathrm{HJ}$, Schroeder MA, Dodd MS, et al. Validation of the in vivo assessment of pyruvate dehydrogenase activity using hyperpolarised ${ }^{13} \mathrm{C}$ MRS. NMR Biomed. 2011;24(2):201-208. https://doi.org/10.1002/nbm.1573

24. Heather LC, Pates KM, Atherton HJ, et al. Differential translocation of the fatty acid transporter, FAT/CD36, and the glucose transporter, GLUT4, coordinates changes in cardiac substrate metabolism during ischemia and reperfusion. Circ Heart Fail. 2013;6(5):1058-1066. https://doi.org/10.1161/ CIRCHEARTFAILURE.112.000342

25. Walsby-Tickle J, Gannon J, Hvinden I, et al. Anion-exchange chromatography mass spectrometry provides extensive coverage of primary metabolic pathways revealing altered metabolism in IDH1 mutant cells. Commun Biol. 2020;3(1):247. https://doi.org/10.1038/s42003-020-0957-6

26. Sowell J, Fuqua M, Wood T. Quantification of total and free carnitine in human plasma by hydrophilic interaction liquid chromatography tandem mass spectrometry. J Chromatogr Sci. 2011;49(6):463-468. https://doi.org/10.1093/chrsci/49.6.463

27. Schroeder MA, Cochlin LE, Heather LC, Clarke K, Radda GK, Tyler DJ. In vivo assessment of pyruvate dehydrogenase flux in the heart using hyperpolarized carbon-13 magnetic resonance. Proc Natl Acad Sci U S A. 2008;105(33):12051-12056. https://doi.org/10.1073/pnas.0805953105

28. Howangyin KY, Silvestre J-S. Diabetes mellitus and ischemic diseases: molecular mechanisms of vascular repair dysfunction. Arterioscler Thromb Vasc Biol. 2014;34(6):1126-1135. https://doi.org/10.1161/ATVBAHA.114.303090

29. Simkhovich BZ, Shutenko ZV, Meirēna DV, et al. 3-(2,2,2-Trimethylhydrazinium)propionate (thp)-a novel $\gamma$-butyrobetaine hydroxylase inhibitor with cardioprotective properties. Biochem Pharmacol. 1988;37(2):195-202. https://doi.org/10.1016/0006-2952(88)90717-4 
30. Rossini AA, Like AA, Chick WL, Appel MC, Cahill GF. Studies of streptozotocin induced insulitis and diabetes. Proc Natl Acad Sci U S A. 1977;74(6): 2485-2489. https://doi.org/10.1073/pnas.74.6.2485

31. Bauman JW. Proteinuria in long term streptozotocin diabetes in rats. Life Sci. 1980;27(22):2121-2124. https://doi.org/10.1016/0024-3205(80) 90493-2

32. Sokolovska J, Isajevs S, Sugoka O, et al. Correction of glycaemia and GLUT1 level by mildronate in rat streptozotocin diabetes mellitus model. Cell Biochem Funct. 2011;29(1):55-63. https://doi.org/10.1002/cbf.1719

33. Statsenko ME, Turkina SV, Belenkova SV, Poletaeva LV, Dudchenko GP. Effects of Mildronate, as a part of combined heart failure therapy, on carbohydrate and lipid metabolism and oxidative stress parameters in patients with type 2 diabetes mellitus. Russ J Cardiol. 2010;82(2):45-51.

34. Liepinsh E, Skapare E, Svalbe B, Makrecka M, Cirule H, Dambrova M. Anti-diabetic effects of mildronate alone or in combination with metformin in obese Zucker rats. Eur J Pharmacol. 2011;658(2-3):277-283. https://doi.org/10.1016/J.EJPHAR.2011.02.019

35. Degrace P, Demizieux L, Gresti J, et al. Fatty acid oxidation and related gene expression in heart depleted of carnitine by mildronate treatment in the rat. Mol Cell Biochem. 2004;258(1/2):171-182. https://doi.org/10.1023/B:MCBI.0000012853.20116.06

36. Abdurrachim D, Woo CC, Teo XQ, Chan WX, Radda GK, Lee PTH. A new hyperpolarized ${ }^{13} \mathrm{C}$ ketone body probe reveals an increase in acetoacetate utilization in the diabetic rat heart. Sci Rep. 2019;9(1):5532. https://doi.org/10.1038/s41598-019-39378-w

37. Miller JJ, Ball DR, Lau AZ, Tyler DJ. Hyperpolarized ketone body metabolism in the rat heart. NMR Biomed. 2018;31(6):e3912. https://doi.org/10. 1002/nbm.3912

38. Ball DR, Rowlands B, Dodd MS, et al. Hyperpolarized butyrate: A metabolic probe of short chain fatty acid metabolism in the heart. Magn Reson Med. 2014;71(5):1663-1669. https://doi.org/10.1002/mrm.24849

39. Abdurrachim D, Teo XQ, Woo CC, et al. Cardiac metabolic modulation upon low-carbohydrate low-protein ketogenic diet in diabetic rats studied in vivo using hyperpolarized ${ }^{13} \mathrm{C}$ pyruvate, butyrate and acetoacetate probes. Diabetes Obes Metab. 2019;21(4):949-960. https://doi.org/10.1111/ dom.13608

40. Chen W, Sharma G, Jiang W, et al. Metabolism of hyperpolarized ${ }^{13} \mathrm{C}$-acetoacetate to $\beta$-hydroxybutyrate detects real-time mitochondrial redox state and dysfunction in heart tissue. NMR Biomed. 2019;32(6):e4091. https://doi.org/10.1002/nbm.4091

41. Bastiaansen JAM, Merritt ME, Comment A. Measuring changes in substrate utilization in the myocardium in response to fasting using hyperpolarized $\left[1-{ }^{13} \mathrm{C}\right]$ butyrate and $\left[1-{ }^{13} \mathrm{C}\right]$ pyruvate. Sci Rep. 2016;6(1):25573. https://doi.org/10.1038/srep25573

42. Yoshihara HAI, Bastiaansen JAM, Karlsson M, Lerche MH, Comment A, Schwitter J. Detection of myocardial medium-chain fatty acid oxidation and tricarboxylic acid cycle activity with hyperpolarized [1-13 C]octanoate. NMR Biomed. 2020;33(3):e4243. https://doi.org/10.1002/nbm.4243

43. Vilskersts R, Liepinsh E, Kuka J, et al. Myocardial infarct size-limiting and anti-arrhythmic effects of mildronate orotate in the rat heart. Cardiovasc Drugs Ther. 2009;23(4):281-288. https://doi.org/10.1007/s10557-009-6179-2

44. Sesti C, Simkhovich BZ, Kalvinsh I, Kloner RA. Mildronate, a novel fatty acid oxidation inhibitor and antianginal agent, reduces myocardial infarct size without affecting hemodynamics. J Cardiovasc Pharmacol. 2006;493-499. https://doi.org/10.1097/01.fjc.0000211732.76668.d2

45. Statsenko ME, Belenkova SV, Sporova OE, Shilina NN. The use of mildronate in combined therapy of postinfarction chronic heart failure in patients with type 2 diabetes mellitus. Klin Med (Mosk). 2007;85(7):39-42.

46. Schonekess BO, Allard MF, Lopaschuk GD. Propionyl L-carnitine improvement of hypertrophied heart function is accompanied by an increase in carbohydrate oxidation. Circ Res. 1995;77(4):726-734. https://doi.org/10.1161/01.RES.77.4.726

47. Liu B, Clanachan AS, Schulz R, Lopaschuk GD. Cardiac efficiency is improved after ischemia by altering both the source and fate of protons. Circ Res. 1996;79(5):940-948. https://doi.org/10.1161/01.RES.79.5.940

48. Miller JJ, Lau AZ, Teh I, et al. Robust and high resolution hyperpolarized metabolic imaging of the rat heart at $7 \mathrm{~T}$ with 3D spectral-spatial EPI. Magn Reson Med. 2016;75(4):1515-1524. https://doi.org/10.1002/mrm.25730

49. Wespi P, Steinhauser J, Kwiatkowski G, Kozerke S. Overestimation of cardiac lactate production caused by liver metabolism of hyperpolarized [1- $\left.{ }^{13} \mathrm{C}\right]$ pyruvate. Magn Reson Med. 2018;80(5):1882-1890. https://doi.org/10.1002/mrm.27197

50. Chen AP, Hurd RE, Schroeder MA, et al. Simultaneous investigation of cardiac pyruvate dehydrogenase flux, Krebs cycle metabolism and pH, using hyperpolarized $\left[1,2-{ }^{13} \mathrm{C}_{2}\right]$ pyruvate in vivo. NMR Biomed. 2012;25(2):305-311. https://doi.org/10.1002/nbm.1749

51. Rider OJ, Apps A, Miller JJJ, et al. Noninvasive in vivo assessment of cardiac metabolism in the healthy and diabetic human heart using hyperpolarized ${ }^{13}$ C MRI. Circ Res. 2020;126(6):725-736. https://doi.org/10.1161/CIRCRESAHA.119.316260

52. Cunningham CH, Lau JYC, Chen AP, et al. Hyperpolarized ${ }^{13} \mathrm{C}$ metabolic MRI of the human heart: initial experience. Circ Res. 2016;11:1177-1182. https://doi.org/10.1161/CIRCRESAHA.116.309769

\section{SUPPORTING INFORMATION}

Additional supporting information may be found online in the Supporting Information section at the end of this article.

How to cite this article: Savic D, Ball V, Holzner L, et al. Hyperpolarized magnetic resonance shows that the anti-ischemic drug meldonium leads to increased flux through pyruvate dehydrogenase in vivo resulting in improved post-ischemic function in the diabetic heart. NMR in Biomedicine. 2021;e4471. https://doi.org/10.1002/nbm.4471 